\title{
OH26.5+0.6: insight into the circumstellar structure of an extreme $\mathrm{OH} / \mathrm{IR}$ star
}

\author{
Sandra Etoka ${ }^{* \dagger}$ \\ School of Physics and Astronomy - The University of Manchester - UK \\ E-mail: Sandra.Etoka@manchester.ac.uk \\ Philip Diamond \\ Jodrell Bank Observatory - The University of Manchester - UK
}

The OH/IR stage is important in the life of an evolved star. This short transitional phase towards the proto-planetary stage is a crucial point in the life of an intermediate- or high-mass star. Indeed, the mass-loss rate when a star reaches this stage is at its highest. The resulting circumstellar shell - interface between the central object and the interstellar medium - is a rich environment of dust and molecules. It is at this stage that asymmetries are detected, even in the outermost part of the circumstellar shell where $\mathrm{OH}$ radicals are formed. $\mathrm{OH} 26.5+0.6$ is an extreme $\mathrm{OH} / \mathrm{IR}$ star with a mass-loss rate of the order of $5 \times 10^{-4} \mathrm{M}_{\odot} / \mathrm{yr}$. It is one of the brightest $\mathrm{OH}$ maser emitters in our Galaxy and it is suspected of experiencing a superwind which emerged less than 150 years ago. MERLIN OH observations at $18 \mathrm{~cm}$ in the 1667, 1665 and 1612-MHz maser line allowed the first combined insight into the geometric and polarimetric structure of the circumstellar material surrounding this object. This work intends to bring a new insight into the structural changes undergone by the circumstellar material of an evolved star when it enters the OH/IR stage.

8th European VLBI Network Symposium

September 26-29, 2006

Toruń, Poland

* Speaker.

${ }^{\dagger}$ A footnote may follow. 


\section{Introduction}

After having left the main sequence, low- and intermediate-mass stars experience a crucial phase in their evolution toward the white dwarf stage: the AGB phase. It is at the very end of this phase that the star, in hardly $10^{-5}$ years, will shed most of its mass through extensive mass loss (up to $10^{-4} \mathrm{M}_{\odot} \mathrm{yr}^{-1}$ ). The precise evolutionary sequence along the AGB of this final stage has not been resolved yet, but $\mathrm{OH} / \mathrm{IR}$ stars are thought to be tracing the ultimate moments of this phase before the star enters the planetary nebula stage.

Surveys confirmed the spherical nature of AGB stars ([11]). Yet, asymmetries such as elliptical shapes or bipolar outflows are commonly observed at the planetary nebula stage ([13]).

Recently, a series of papers investigated the polarimetric structure in the intermediate and outermost parts of the circumstellar shell of evolved stars ([8], [2], [6], [12]). Although the origin and evolution of the magnetic field is not well understood yet and is currently a matter of debate, this series of papers has shown the importance of the magnetic field in shaping the circumstellar material.

$\mathrm{OH} 26.5+0.6$ is an extreme $\mathrm{OH} / \mathrm{IR}$ star with an estimated initial mass of $8 \mathrm{M}_{\odot}$ and a current mass-loss rate of the order of $5 \times 10^{-4} \mathrm{M}_{\odot} / \mathrm{yr}$. Also, this object is suspected of experiencing a superwind which emerged less than 150 years ago [9]. This makes $\mathrm{OH} 26.5+0.6$ a very particular and important object in terms of stellar evolution: a junction object between an intermediate- and a high-mass evolved object on the verge of leaving the AGB towards the planetary nebula phase.

\section{Observations}

The observations were made on the $12^{\text {th }}$ December 1993 at 1612, 1665 and $1667 \mathrm{MHz}$ using the 8 telescopes of MERLIN available at that time (namely Defford, Cambridge, Knockin, Wardle, Darnhall, Mk2, Lovell \& Tabley) giving a maximum baseline of $217 \mathrm{~km}$ and a resolution of 0.17 arcsec. They were carried out in full polarization mode in order to retrieve all four Stokes parameters and determine the magnetic field as well as the geometric structure at the location of the $\mathrm{OH}$ maser emission.

\section{Results}

The intensity and the profile shape retrieved from the final map were compared with the singledish spectra obtained in 1973 ([1]). They show that we recovered most of the signal. Therefore, the fraction of emission lost in possible extended structure is small.

\subsection{Maser emission extent}

The velocity-averaged images in Stokes I for the 1612, 1667 and 1665-MHz maser emission are presented, from left to right, in Fig 1 . The total extent of the maser emission at $1612 \mathrm{MHz}$ is about 5 arcsec which corresponds to a linear extent of $7000 \mathrm{AU}$ at $1.4 \mathrm{kpc}$, the estimated distance of $\mathrm{OH} 26.5+0.6$. At $1667 \mathrm{MHz}$, the emission in the south of the shell extends beyond that of the $1612 \mathrm{MHz}$ by nearly 1 arcsec. The 1612-MHz emission reveals the ellipsoidal nature of the $\mathrm{OH}$ 


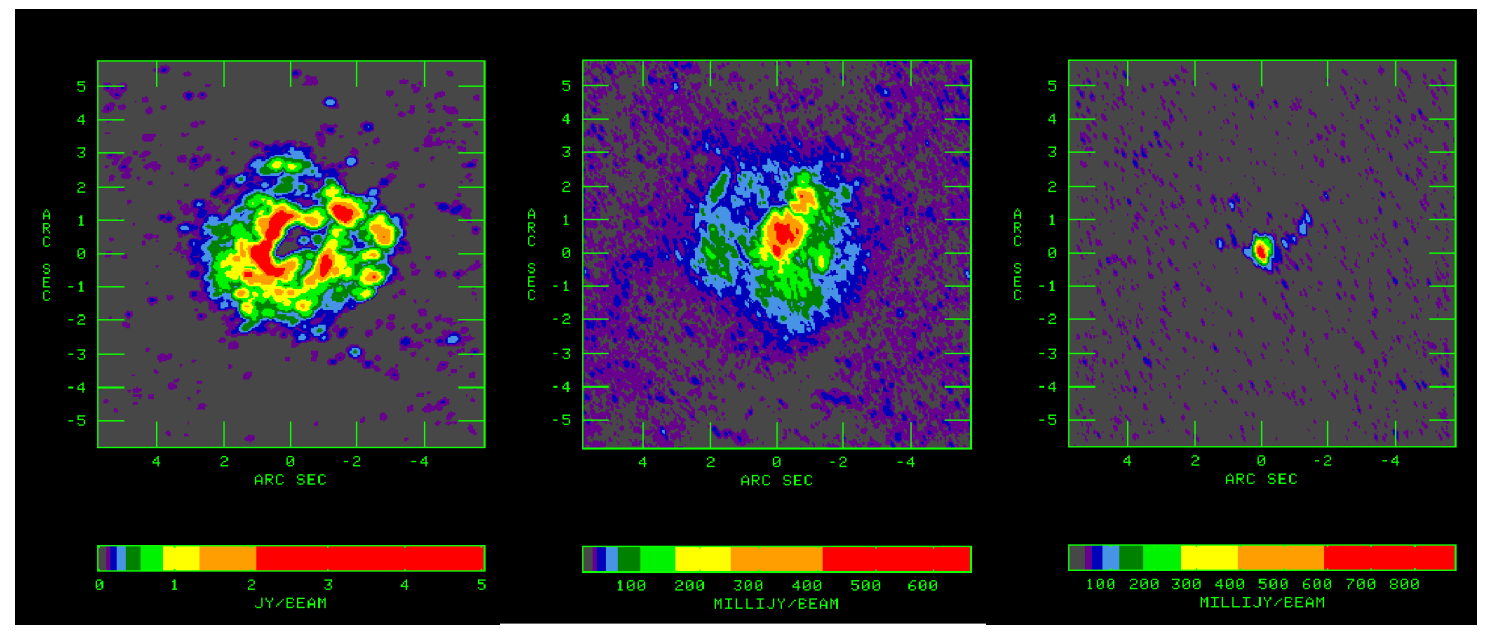

Figure 1: Velocity-averaged emission in Stokes I. Left panel: $1612 \mathrm{MHz}$, excluding the strong central counterpart. Central panel: $1667 \mathrm{MHz}$. Right panel: $1665 \mathrm{MHz}$.

masing shell. The $1665-\mathrm{MHz}$ central core emission lies within an area of less than 1.5 arcsec. A filamentary structure is clearly visible in the $\mathrm{N}-\mathrm{NW}$ part of the shell extending over $\simeq 2$ arcsec. Including this emission and the very faint maser spots observed east, the total extent of the emission is still less than $4 \operatorname{arcsec}$ and gives a dramatically different picture than that of the $1667 \mathrm{MHz}$ emission.

\subsection{Maser spot distribution}

The AIPS task SAD was used to identify maser components in the individual channel maps. At $1665 \mathrm{MHz}$ the simplicity of the maps were such that a $3 \sigma$ threshold was taken to retrieve the maser components. At 1612 and $1667 \mathrm{MHz}$, the maps being complex, a more stringent selection has been applied for retrieving the components. A component has been accepted only if its flux density was greater than $4 \times$ rms noise of a given channel (or greater than $10 \times$ rms noise in very complex regions). Left and right panels of Fig. 2 show the corresponding maser spot distributions at 1667 and $1612 \mathrm{MHz}$ respectively. In both lines, the south part of the shell clearly shows the domination of blue-shifted emission.

The information concerning the magnetic field structure at the location of the $\mathrm{OH}$ maser emission is displayed in Fig. 2 2 via the vectors of polarization showing the electric field structure. These are shown for the maser spots exhibiting polarized emission greater than $3 \sigma$. At $1667 \mathrm{MHz}$, only the 2 strongest red-shifted maser spots fulfil this criterion. At $1612 \mathrm{MHz}$, over a third of the maser spots (106 out of the 277) fulfil this criterion. It reveals a highly ordered polarization vector field. Overall, the polarization vectors show a mixture of radial (in the northern part of the shell) and tangential (in the southern part) distributions separated by an axis with a projected angle of about $20-30^{\circ}$ on the sky plane.

\subsection{Radial velocity distribution}

The $V=\mathrm{f}(\theta)$ distributions at 1667 and $1612 \mathrm{MHz}$ are shown in Fig. 3 in the left and right panels 

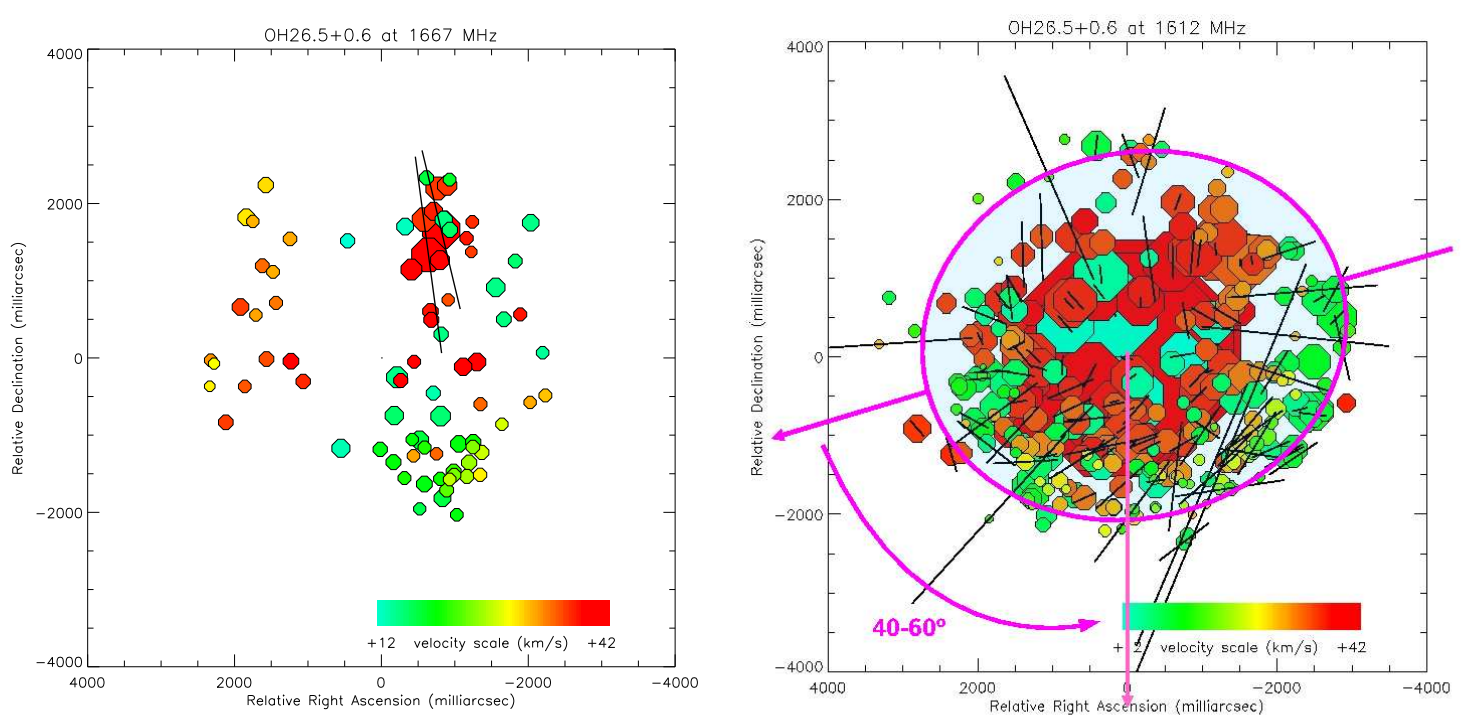

Figure 2: Distribution of the maser spots. The symbols are proportional to the maser spot intensity and the colour scale indicates their velocity. Also plotted are the polarization vectors associated with each maser spot showing a polarized light greater than $3 \sigma$. Left panel: at $1667 \mathrm{MHz}$. Right panel: at $1612 \mathrm{MHz}$ on which is overlaid a model explaining both the geometric and polarimetric structures observed (cf. text).

respectively. The best fits for the lower and higher boundaries of the radial velocity distribution are also displayed in the figures. These correspond to a simple model where a spherical thin shell expands radially at a constant velocity. This simple model allows us to infer the $\mathrm{OH}$ masing region thickness to about 1.5-2 arcsec, and that acceleration is still taking place in the outer part of the circumstellar envelope. Both distributions lead to similar results. But they also show that clearly this model fails to represent the actual radial velocity distribution observed. Overlaid on the 1612$\mathrm{MHz}$ velocity distribution (right panel of Fig. 3) are two schematic models consistent with the distribution observed. These more sophisticated models are obtained in the case of a prolate shell tilted about $45^{\circ}$ (dotted line) to $65^{\circ}$ (solid line) to the line of sight expanding radially (cf. [3]).

\section{Discussion and conclusion}

At $1667 \mathrm{MHz}$, the emission in the south of the shell extends beyond that of the $1612 \mathrm{MHz}$ by nearly 1 arcsec. This is an interesting result since main-line emission (i.e., 1665 and $1667 \mathrm{MHz}$ ) is expected to be internal to that of the 1612-MHz satellite line due to competitive gain which leads to the quenching of the main-line inversion when the 1612-MHz line reaches saturation (沺). The extension of the $1667-\mathrm{MHz}$ emission beyond that of the $1612 \mathrm{MHz}$ requires deviation from the standard model ([4]).

Zeeman's pairs were identified allowing us to estimate a magnetic field strength of 3-4 mG at the location of the $\mathrm{OH}$ maser shell (i.e., $7000 \mathrm{AU}$ ). A similar value, also at a similar distance was found for the evolved supergiant star NML Cyg ( $3 \mathrm{mG}$ at a distance of $10^{4} \mathrm{AU}$ [6]). 

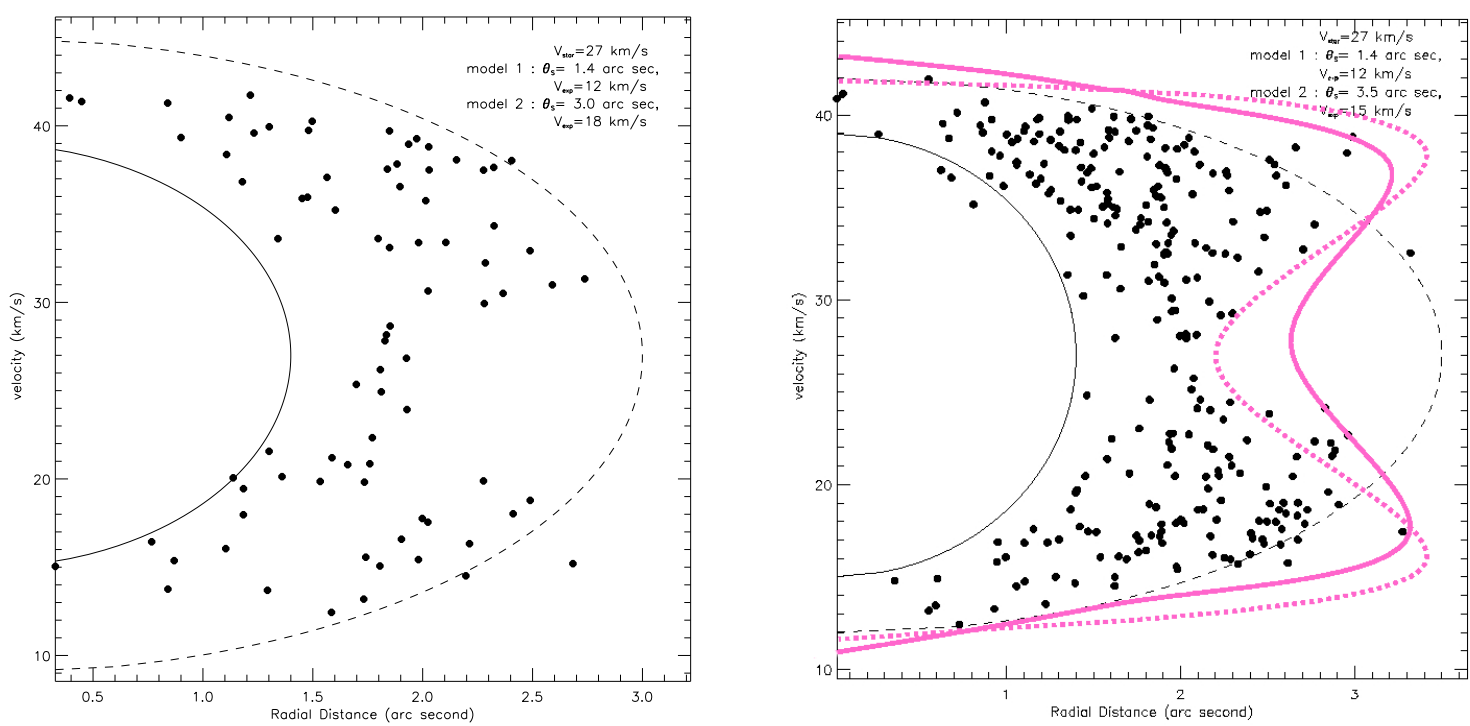

Figure 3: The radial velocity distributions. Overlaid are a series of models discussed further in the text. Left panel: at $1667 \mathrm{MHz}$. Right panel: $1612 \mathrm{MHz}$.

Fig. 2 presents superimposed on top of the maser spot and polarization vector distributions: 1) the projected axis segregating the radial and tangential vectors of polarization and 2) the ellipse that describes best the maser spot distribution observed. The polarization structure observed is consistent with a poloidal magnetic field inclined by $40-60^{\circ}$ to the line of sight. Such a configuration accounts for the change of orientation of the polarization vectors between tangential and radial as observed here ([5], [10]).

The projected axis on the line of sight separating the tangential and radial distribution of the vectors of polarization is aligned with the major axis of the geometrically ellipsoidal maser emission (cf. Fig. 2). This latter projected ellipse and the velocity distribution of the maser spots as observed in Fig. 3 are indeed expected if the actual shell geometry is a prolate spheroid tilted about $45-65^{\circ}$ to the line of sight ([3]).

These combined results reveal that there is a definite correlation between the magnetic field orientation and the geometrical structure of the circumstellar envelope.

\section{References}

[1] Andersson C., Johansson L.E.B., Goss W.M., Winnberg A.,Ngugen-Quang-Rieu, 1974, A\&A 30, 475

[2] Bains I., Gledhill T.M., Yates J.A., Richards, A.M.S., 2003, MNRAS 338, 287

[3] Bowers P.F., 1991, ApJS 76, 1099

[4] Collison A.J., Nedoluha G.E., 1995, ApJ 442, 311

[5] Elitzur M., 1992, Astronomical Masers (Dordrecht:kluwer)

[6] Etoka S., Diamond P.J., 2004, MNRAS 348, 34

[7] Field D., 1985, MNRAS 217, 1 
[8] Imai H., Obara K., Diamond P.J., Omodaka T., Sasao T., 2002, Nature 417, 829

[9] Justtanont K., Skinner C.J., Tielens A.G.G.M., Meixner M., Baas F., 1996, ApJ 456, 337

[10] Kemball A.J., Diamond P.J., 1997, ApJ 481, L111

[11] Sahai R., 2002, Rev. Mex. Astron. Astrofis. Ser. Conf. 13, 133

[12] Vlemmings W.H.T., van Langevelde H.J., Diamond P.J., 2005, A\&A 434, 1029

[13] Zijlstra A., Lagadec E., Matsuura M., Chesneau O., Etoka S., 2006, The Messenger vol. 124, p. 21 